\title{
POINT/COUNTERPOINT
}

Suggestions for topics suitable for these Point/Counterpoint debates should be addressed to the Moderator: William R. Hendee, Medical College of Wisconsin, Milwaukee: whendee@mcw.edu.Persons participating in Point/Counterpoint discussions are selected for their knowledge and communicative skill. Their positions for or against a proposition may or may not reflect their personal opinions or the positions of their employers.

\section{High energy electron beams shaped with applied magnetic fields could provide a competitive and cost-effective alternative to proton and heavy-ion radiotherapy}

\author{
Frederick D. Becchetti \\ Physics Department, University of Michigan, Ann Arbor, Michigan 48109 \\ (Tel: 734/764-1598; E-mail: fdb@umich.edu) \\ Janet M. Sisterson ${ }^{\text {a) }}$ \\ Massachusetts General Hospital, Northeast Proton Therapy Center, Boston, Massachusetts 02114 \\ (Tel: 617/724-1942; E-mail: jsisterson@partners.org) \\ William R. Hendee, Moderator
}

(Received 29 July 2002; accepted for publication 29 July 2002; published 30 September 2002)

[DOI: $10.1118 / 1.1510453]$

\section{OVERVIEW}

Work at several institutions has demonstrated that intense magnetic fields can be used to confine and shape high-energy electron beam-dose profiles. It is conceivable that this approach might compete effectively with hadron therapy. However, the technique gives rise to several technical challenges, some of which may be insurmountable. Whether the technique has enough potential to be pursued as a possible approach for radiation therapy is the subject of this month's point/counterpoint debate.

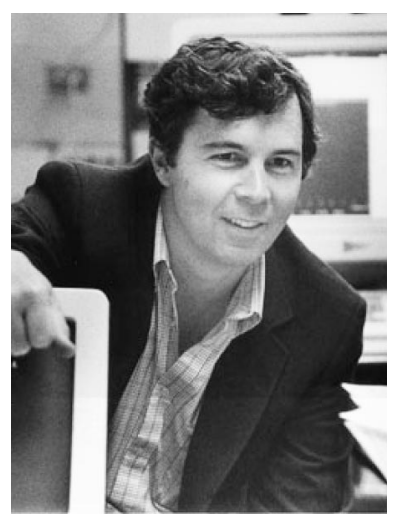

Arguing for the Proposition is Frederick D. Becchetti, Ph.D. Dr. Becchetti has worked primarily in accelerator-based nuclear physics including the application of large superconducting magnets to nuclear reaction studies and recently, application of the latter to radiation oncology. He has served on the Board of Editors of Review of Scientific Instruments (1999-2001) and was associate program chair for the recent Symposium on Radiation Measurements and Applications held in Ann Arbor, Michigan, May 21-23, 2002. Dr. Becchetti has chaired or co-chaired a number of Ph.D. thesis committees dealing with medical imaging, radiation oncology or related areas in nuclear medicine.

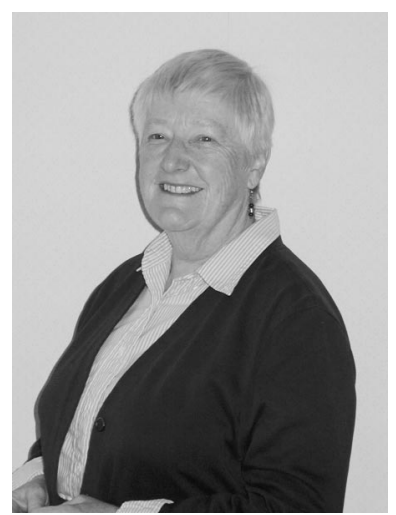

Arguing against the Proposition is Janet M. Sisterson, $\mathrm{Ph}$.D. Dr. Sisterson trained as a Medical Physicist in London following receipt of a Ph.D. in high-energy physics from the University of London. She spent 25 years at the Harvard Cyclotron Laboratory where she helped develop many of the techniques used worldwide in proton radiation therapy. In 1998 she moved to the Northeast Proton Therapy Center, Department of Radiation Oncology at the Massachusetts General Hospital. She holds a joint appointment as Assistant Professor in the Department of Radiation Oncology at Harvard Medical School. She is the principal investigator on a NASA grant to measure proton and neutron cross sections needed for cosmic-ray studies.

\section{FOR THE PROPOSITION: Frederick D. Becchetti, Ph.D.}

\section{Opening Statement}

Calculations $^{1}$ and measurements using phantoms ${ }^{2,3}$ have shown that the radiation dose profile from high-energy electron beams typically used in radiotherapy can be confined using high (viz., a few tesla) magnetic fields. The large penumbra associated with scattering of electron beams in tissuelike material is thereby greatly reduced. In addition, for magnetic fields parallel to the initial beam direction, additional, 
3D focusing of the electron beam may be obtained. The net effect is a greatly enhanced dose near the end of the electrons' range. The dose profile resembles a degraded Bragg curve. ${ }^{2,3}$ This feature could be exploited in conformal radiotherapy to furnish an alternative to proton or heavy-ion radiotherapy, at least for certain types of tumors, and to provide an economical, on-site electron-beam therapy facility for many hospitals. High-energy electron accelerators (e.g., linacs and microtrons) and electron-beam gantries are affordable by many hospitals. This is in contrast to current proton and heavy-ion radiotherapy units, which are large, costly facilities located far from most hospitals and hence not practical for widespread use, especially when fractionated doses are needed. Many of the technical problems have been solved, particularly the use and efficient operation of largebore superconducting magnets and such magnets are in routine use in MRI units at most large hospitals. Such magnets can be designed, if needed, to allow rotation with a treatment gantry. ${ }^{4}$ Recently mechanical coolers have been utilized which can further simplify a gantry-mounted system, and nonmagnetic treatment gantries and tables are feasible. Further research and development appears warranted and if successful could lead to animal and, eventually, human clinical trials in the near future.

\section{Rebuttal}

It is not necessarily claimed that electrons confined by a magnetic field are preferable to high-energy protons (or heavy ions) for many types of radiotherapy. Instead, as noted, the high cost of high-energy ion accelerators, gantries, radiation shielding and buildings has greatly limited the number of patients that can be treated with protons. For some patients, magnetically-confined electron-beam therapy may in the future provide a viable alternative to protons at lower cost. The experiments cited in Refs. 2 and 3 utilized a hospital-based "table top" $50 \mathrm{MV}$ electron racetrack microtron. $100 \mathrm{MV}$ electron microtrons used for physics research are similarly in operation. Providing an accurate 3D focus for stereotactic treatment with electrons is perhaps the major challenge. As noted in the opening statement, largebore superconducting magnets (e.g., $1 \mathrm{~m}$ diam. bore $\times 1 \mathrm{~m}$ long $B \geqslant 5 T$ ) are feasible, and can be gantry mounted. Such magnets are presently used in research and industrial applications as well as in medical applications (high-field MRI). Thus much of the needed engineering has already been done. The key issue is to demonstrate that magnetically-confined electron-beam radiotherapy may have advantages over other modalities, even $\mathrm{x}$-ray IMRT in certain circumstances. ${ }^{1-3,5}$ However, this issue can be addressed definitively only by further research including realistic treatment simulations and animal clinical trials.

\section{AGAINST THE PROPOSITION: Janet M. Sisterson, Ph.D.}

\section{Opening Statement}

Computer simulations and some experiments show that intense magnetic fields can be used to delineate the geometry and control the dose distribution of high-energy electron beams. For this reason, electron beams have been proposed as a cost-effective alternative to heavy ion beams, with a similar therapeutic benefit. Simulations and experiments have been conducted in an attempt to overcome the inherent limitations of electrons, all due to the small electron mass, and to mimic, by external means, the inherent properties of ion beams. The goal of these experiments has been to identify applications where electron beams can be equally or preferentially used to treat specific tumor sites.

The inherent physical properties of ion beams are used to advantage in radiation therapy. These properties include: (1) little penumbral scattering as the beam penetrates tissue; (2) a finite range in tissue, accurately controlled by the beam energy; (3) a very sharp fall-off in the distal edge of the dose distribution; and (4) a maximum rate of energy loss near the end of range: "the Bragg peak effect." These properties are used to achieve dose distributions that are uniform over large target volumes, with a sharp penumbra in all directions perpendicular to the beam and along the axis. The resulting dose distributions conform closely to the target volumes, leading to maximum sparing of adjacent normal tissues and sensitive structures.

Electrons, as continuously ionizing particles, share some of the properties of heavy ions. In electron beams, however, the benefit of these properties is lost because of the extreme scattering of individual electrons. Intense magnetic fields can limit the scattering effects to a significant degree. Still, they do not allow electrons to achieve the properties of an ion beam Bragg peak or the dose distributions attainable by summation of such peaks. For example, the proposed magnetic fields focus primarily in one dimension. This implies that any magnetic focusing technique will require much more sophisticated, three-dimensional control of the magnetic focus inside the patient. In addition, electron energies up to $100 \mathrm{MeV}$ may be needed to cover the full range of therapeutic applications. The construction of a device that delivers highenergy electron beams with a precisely controlled threedimensional magnetic focusing lens may prove to be an insurmountable, and certainly not cost-effective, engineering task.

In contrast, intensity and energy modulated ion therapy is already a reality, and permits precise control of dose deposition in volumes as large as $20000 \mathrm{~cm}^{3}$ with a resolution of $0.125 \mathrm{~cm}^{3}$. The technology for delivering these fields is proven and uses off-the-shelf magnetic devices and control techniques. Field delivery is accurately controlled in both time and space, a critical benefit when considering patientspecific concerns such as organ motion.

In summary, one-dimensional magnetic control of electron fields may improve the therapeutic gain compared with "conventional" electron fields. Such control is no match for intensity modulated $\mathrm{x}$-ray therapy, conventional ion therapy, or intensity modulated ion therapy. There is simply not a clear rationale to justify pursuit of such a nontrivial and expensive engineering task as magnetic field-controlled electron beam therapy. 


\section{Rebuttal}

Our colleague claims that electron beams, modified with (strong) magnetic fields, can be delivered and controlled in a clinical setting to produce therapeutic dose distributions that might be an alternative to proton or heavy ion radiotherapy. Such an alternative is deemed desirable due to the high cost of a proton or ion facility, and the implied geographic separation of such a facility from a hospital. We believe there are several misconceptions in this position. First, the Harvard Cyclotron Laboratory/Massachusetts General Hospital collaboration (HCL), with over 9,000 patients treated, shows that fractionated proton radiotherapy can be successfully accomplished using a facility located about three miles from the hospital campus. Second, it is true that proton and ion therapy were pioneered at existing accelerator facilities, often under many constraints. However, there are currently many hospital on-site proton centers treating patients, under construction, or planned. Such facilities are expensive but can still be cost-effective. For example, the cost of such a facility is amortized over the 30-year lifetime of a cyclotron. (Of note, the HCL operated for 50 years!) Over that lifetime, a conventional clinic, albeit a large one, would purchase about 12 linear accelerators comparable to the multiple treatment rooms in a proton therapy facility. This analysis cer- tainly reduces the gap in spending between a conventional clinic and a clinic that also provides proton therapy. Finally, we again stress that the predicted electron dose distributions are not comparable to conventional, energy-modulated, proton Bragg peaks, and certainly not to intensity and energy modulated proton beams or photon IMRT.

${ }^{a)}$ Dr. Sisterson's section was written with the assistance of Hanne M. Kooy, Ph.D., Associate Director Northeast Proton Therapy Center, Massachusetts General Hospital and Associate Professor, Department of Radiation Oncology, Harvard Medical School, Boston, MA.

${ }^{1}$ A. F. Bielajew, "The effect of strong longitudinal magnetic fields on dose deposition from electron and photon beams," Med. Phys. 20, 1171-1179 (1993), and references within.

${ }^{2}$ D. W. Litzenberg, A. F. Benedick, D. L. McShan, T. W. O’Donnell, D. A. Roberts, F. D. Becchetti, A. F. Bielajew, and J. M. Moran, "An apparatus for applying strong longitudinal magnetic fields to clinical photon and electron beams," Phys. Med. Biol. 46, N105-N115 (2001).

${ }^{3}$ F. D. Becchetti, D. W. Litzenberg, J. M. Moran, T. W. O’Donnell, D. A. Roberts, B. A. Fraass, D. L. McShan, and A. F. Bielajew, "Magnetic confinement of radiotherapy beam-dose profiles," Proceedings of Cyclotrons and Their Applications 2001, Sixteenth International Conference, edited by F. Marti (AIP, Melville, 2001), pp. 44-46.

${ }^{4} \mathrm{H}$. Blosser et al., "Superconducting cyclotron for medical applications," IEEE Trans. Magn. 25, 1746-1754 (1989).

${ }^{5}$ J. Chu, W. Hsi, and L. Reiffel, "Potential application of transverse magnetic field in x-ray therapy," Med. Phys. 28, 1277 (abstract) (2001). 